\title{
Quality Assessment of Information on Oral Cancer Provided at Arabic Speaking Websites
}

\author{
Mohammed Sultan Alakhali ${ }^{1,2 *}$
}

\begin{abstract}
Background: Patients often use Inernet to explore information about their health and disease. This study aimed to evaluate the quality of information on oral cancer provided at Arabic websites. Material and Method: The translated Arabic keywords of "oral cancer" and treatment of oral cancer" and 6 search engines were used. The top 100 websites were selected. Irrelevant and duplicates websites were excluded. To evaluate the quality of websites and their information, DISCERN, HON, and JAMA benchmarks were used. Results: The majority ( $n=64,74.4 \%$ ) of websites were founded by profit organizations. The mean overall rating was 2.23 (1.6) out of 5 and the median overall rating was 1 (1-3) based on DISCERN. According to HON, only $4.7 \%$ of the websites achieved a high score $(\geq 75)$ and $37.2 \%$ of the websites gained a low score $(<50)$. Based on JAMA benchmarks, $15 \%$ of the websites did not fulfill any criteria and only $2 \%$ of them fulfilled the four criteria proposed by the JAMA benchmarks. Conclusion: This study suggested the poor quality of web-based Arabic information on oral cancer. It is recommended to develop a websites based system by which enable to the Arabic websites related to oral cancer know their shortfalls, therefore, improve their quality according to evaluation tools which will ensure finding reliable data from the websites.
\end{abstract}

Keywords: Internet- oral cancer- Arabic information- websites- quality evaluation

Asian Pac J Cancer Prev, 21 (4), 961-966

\section{Introduction}

The Internet has become one of main information to search for health and disease. A lot of patients incline to browse websites to clarify their health-related concerns, even before consulting the specialist. In a previous study, it was reported that $80 \%$ of US population were searching for online health information, including treatment methods (Castleton et al., 2011). In Saudi Arabia, among 344 diabetic patients, only $39 \%$ were Internet users, of whom $71.6 \%$ used the Internet for seeking health-related information (Jamal et al., 2015). In addition, it was found that more than three-quarters of the Arab participants (79\%) did not use the Arabian health websites (Al Huziah et al., 2009). Mouth cancer is the $6^{\text {th }}$ prevalent cancer and considered among the ten top leading cause cancer related death in the world with an estimated overall annual incidence of nearly 443,000 new cases and 241,450 deaths worldwide (Forman et al.; Ferlay et al., 2015). Most of the new oral cancer cases are diagnosed in the developing world (Warnakulasuriya, 2009). The Middle East and North Africa region is constituted of 23 states, with a population of over 448 million people speaking Arabic. The incidence of cancer in the Middle East and North Africa region is on the increase, and it is currently the 4th leading cause of death (Organization and Organization,
2009). The incidence rates of oral cancer in these countries for females and males were 1.8 and 2.6, respectively, per 100,000 per annum in 2015 (Ferlay et al., 2015). Chewing habits, such as chewing Shamma, tobacco, and khat are extensively used in Arabic countries (Al-Ak'hali et al., 2017). There is a strong relationship between chewing Shamma and oral cancer (Basha et al., 2019).

The diagnosis of oral cancer may cause distress for the patient because the threat of life caused by oral cancer such as disability and pain and it is an effect on the physical and psychosocial status of the individual. Living with such potentially malignant disease is associated with modifiable risk factors such tobacco, excessive alcohol consumption and the use of betel quid, often requires effective health motivation and behavior changes, which demand that an individual has an awareness of their disorders and is provided with the data and solutions necessary to initiate and maintain these behavior changes (Schwarzer and Fuchs, 1995; Warnakulasuriya, 2010). Many researchers have evaluated the impact of online information on health behaviors changes, including disease treatment and tobacco smoking cessation (Strecher, 1999; Portnoy et al., 2008). There is huge information on oral cancer at Arabic websites. However, to the best of our knowledge, this is the first study that evaluated the quality and content of web-based Arabic information on oral cancer through 
evaluation of all Arabic internet websites have shown the original information related to oral cancer.

\section{Materials and Methods}

The the searching and identification of websites were done on 17 Jun 2019. Before conducting browsing, the author erased the cookie information and the browser. Browsing was done using incognito mode to prevent bias arising from preceding searches.

\section{Searching and selecting websites}

The most six popular search engines in Arabic, including "Google (http://www.google.com)", "Yahoo (http://www.yahoo.com)", "Bing (http://www.bing.com)", "Ask (http://www.ask.com)", "Aol. (http://www.aol. com)", and "MSN (http://www.msn.com)" were used to identify the websites (Lebbos et al., 2014). The translated Arabic keywords of "oral cancer" and treatment of oral cancer" were used during the searches. The first 10 pages from each search engine were browsing visited within 24 hours to avoid any changes. Therefore, the first 100 consecutive websites identified from each engine were browsed using the first key word in Arabic language that was "oral cancer". Then, similarly the first ten pages were browsed again using the second keyword in Arabic language, "treatments of oral cancer". The default settings were kept and any advanced search options were avoided.

\section{Exclusion criteria}

At first, the repeated websites were eliminated. Then, extracted websites were excluded if:

a. did not use the Arabic language to present the information

b. mentioning oral cancer just by the hint only, exclusive audio, or visual-based

c. complete scientific articles or textbook

d. they were found to copied the report from another site

e. they included banner advertisements, sponsored links, or discussion forums

f. they denied direct access through keywords used

g. they contained no information on oral cancer

h. Workshops

\section{Inclusion criteria}

Websites that had a free entree, without a password requisite, presented in Arabic language, and provided information on oral cancer were involved.

\section{Evaluation criteria}

Websites that fulfilled the inclusion criteria were evaluated by two examiners independently and a common agreement was reached. The quality of the websites was evaluated using Discern plus Assessment (Charnock et al., 1999), JAMA Benchmarks (Silberg et al., 1997), and HONs assessment tools (Boyer et al., 2011). Intra and Inter-examiner calibration was done before assessing the quality of websites. The DISCERN, HON, and the JAMA benchmarks were selected as the validated tools to evaluate the quality of the medical websites. DISCERN is a questionnaire that provides users with a valid way of evaluating the quality of written information on different treatments for a health disorder (Charnock et al., 1999). This questionnaire includes 16 questions, divided into 3 sections. Questions 1-8 address the trustworthiness of the publication, which help to consider whether it can be trusted as a source of data about selected therapy, questions 9-15 focus on therapy options, and question 16 corresponds to the overall quality score at the end of the evaluation (Charnock et al., 1999). Each question is scored using a five-point Likert scale ( 1 indicates a poor quality and 5 a good quality). One expert (dentist) evaluated the consistency in marking websites using DISCERN. The JAMA benchmarks published by the Journal of the American Association include four standards: authorship, attribution, currency, and disclosure (Silberg et al., 1997). $\mathrm{HON}$ is a non-profit foundation that aims to assess the transparency and quality but not the precision of web-based health data. HON includes eight criteria: authority, complementarity, confidentiality, justifiability, attribution, financial disclosure, transparency, and advertising policy. HON page assistances the evaluator to evaluate the credibility, transparency, and quality of a health website by guiding the evaluator through questions related to the HON code principles and the Europe Quality Criteria for Health-related Websites. After replying a series of questions, the site will be given a grade in percent and indications regarding its level of production quality and transparency (Boyer et al., 2011). Two trained evaluators, both of whom were dentists, conducted the identification and evaluation of the websites.

Data were analyzed using SPSS (version 17) and were expressed in terms of frequency and percentages.

\section{Results}

The search process by using both keywords resulted in thousands of websites (Table 1). After eliminating the duplicates, only 184 sites were selected out of 600 websites (top 100 links from each search engine and keywords). In accordance with our exclusion criteria, 98 sites were then excluded (Figure 1). Therefore, 86 sites which met our inclusion criteria were included for further analysis (Figure 2).

\section{Search results obtained by each search engine}

For all searches, Google introduced the highest related content; 87 sites, with less duplicating websites, compared to other search engines (Figure 2). Yahoo and Bing revealed the same number of sites; 20 sites for each engine with 61 and 75 duplicated sites, respectively. Ask, Aol, and MSN introduced 12, 21, and 24 related sites with 77, 78, and 90 duplicating websites, respectively (Figure 2). Highest excluded content was from MSN; 24 sites, followed by Aol; 20 sites. According to excluding criteria, 13, 16, 19, and 6 sites were respectively excluded from searches obtained from Google, Yahoo, Bing, and Ask introduced (Figure 2).

According to funding background, the profit organization websites hosted 64 sites $(74.4 \%)$ and non-profit organizations hosted 22 sites $(25.6 \%)$. 
Table 1. Summary of Websites Classification According to Funding Background and Affiliation

\begin{tabular}{llcc}
\hline \multicolumn{1}{c}{ Sub-classification } & \multicolumn{2}{c}{ Distribution } & $\%$ \\
\hline Profit-based & \multicolumn{1}{c}{ Profit } & Frequency & 74.4 \\
& Non-profit & 64 & 25.6 \\
Profit-based expanded & Profit/Newspaper & 22 & 17.4 \\
& Profit/Social & 15 & 46.5 \\
& Profit/Medical Center & 40 & 10.5 \\
Affiliation-based & Non-profit & 9 & 25.6 \\
& Medical Center & 22 & 10.5 \\
& Newspaper & 9 & 17.4 \\
& Forum & 15 & 10.5 \\
& Cultural & 9 & 34.9 \\
\hline
\end{tabular}

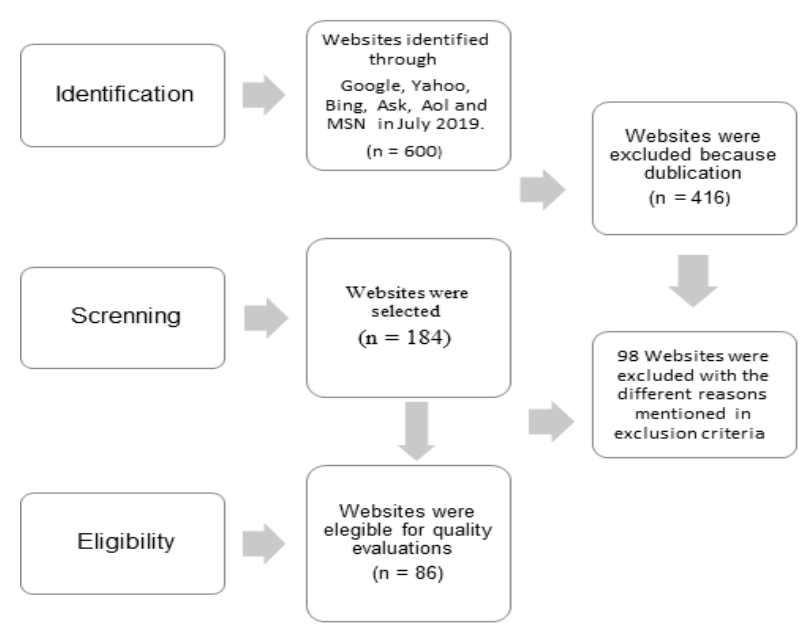

Figure 1. Diagram Shows the Inclusion and Exclusion of Websites for Evaluation

According to the affiliation, the websites were divided to the following categories: Medical centers and clinics:
9 sites (10.5\%), Newspapers: 15 sites $(17.4 \%)$, forums: 9 sites (10.5\%), Cultural sites: 30 sites (34.9\%), and Medical sites: 23 sites $(26.7 \%)$ (Table1).

\section{Quality assessment}

\section{DISCERN}

Based on the overall quality score and using DISCERN, the quality assessment of the 86 identified websites showed a mean overall rating of 2.23 (1.6) out of 5 and a median overall rating of 1 (1-3) (Table 2).

The question about explicit aims scored median of 2 (1-4) and mean of 2.68 (1.39) out of 5. Most of the websites revealed the date of the information with a median of 3 (1-4) and mean of 2.63 (1.27), however a few number of websites have revealed the source of the information a median of 1 (1-1) and mean of 1.51 (1.11) out of 5 (Table 2).

Majority of the websites had given a correct account of how the treatment of oral cancer works a median of 3 (1-4). All alternatives correctly described a median of 2 (1-3). Benefits and risks of treatment were correctly

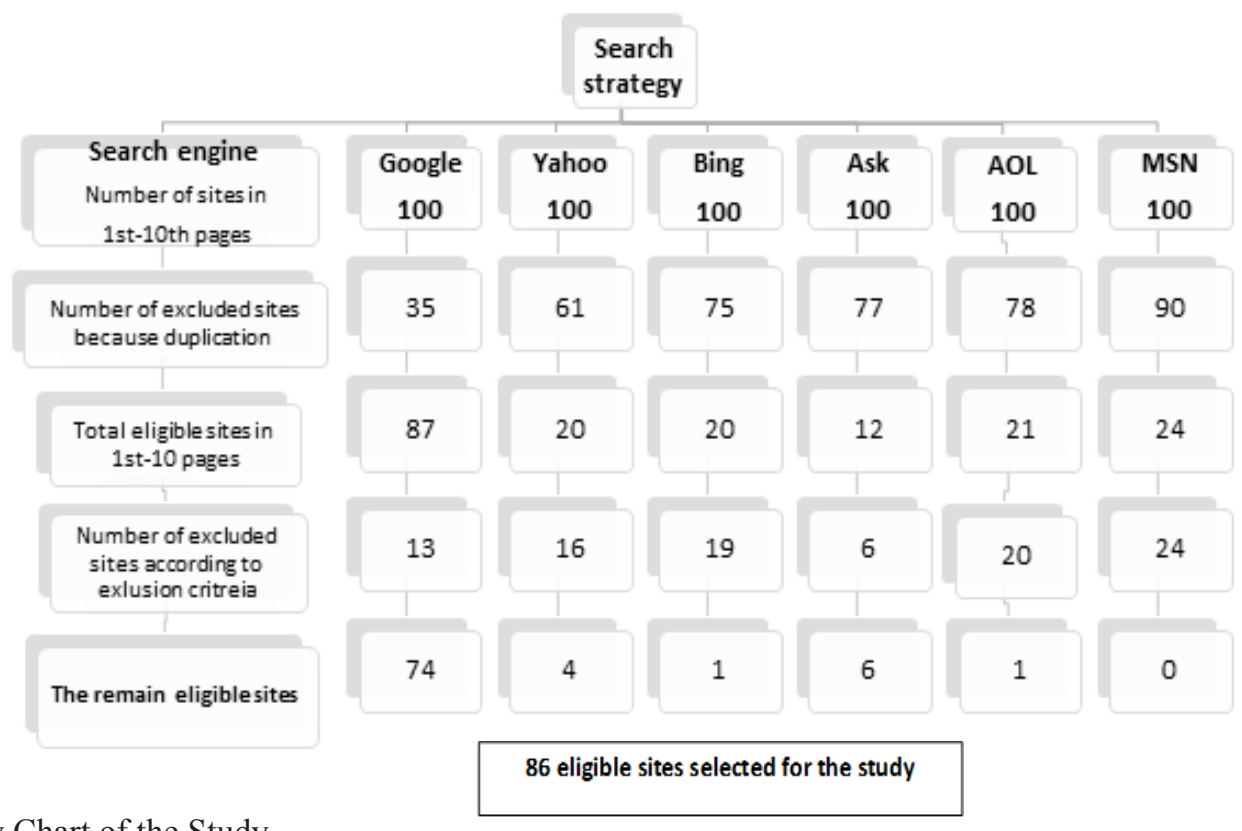

Figure 2. Flow Chart of the Study 


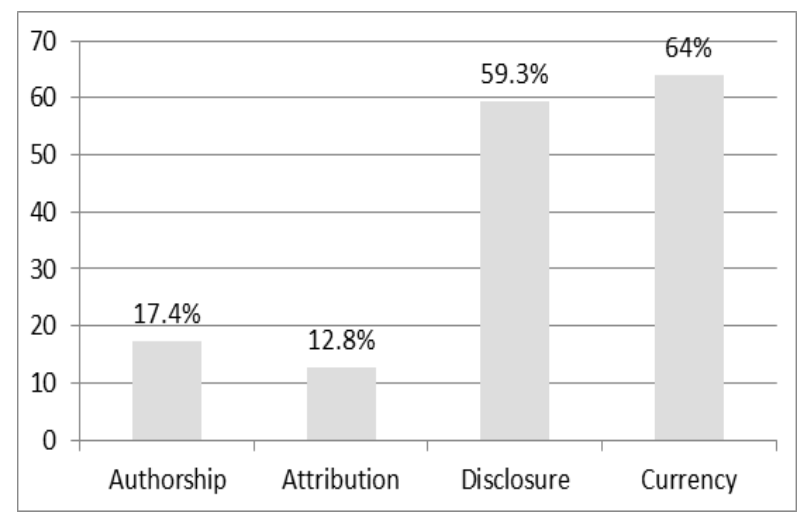

Figure 3. Shows Evaluation the Quality of the Medical Websites Using the JAMA Benchmarks

given a median of 2.5 (1-3) and 1 (1-3), respectively. The effects on the quality of life due to oral cancer were mentioned a median of 2 (1-3) while details regarding the Shared decision for oral cancer patients were mentioned a median of 2 (1-3) (Table 2).

The average measure for intra examiner and inter-examiners assessment of DISCERN were at 0.823 and $0.843(\mathrm{P}<0.001)]$ respectively. The score between 0.75 and 0.90 demonstrates good reliability (Koo and Li, 2016).

\section{JAMA Benchmarks}

The number and percentage of websites per obtained JAMA benchmark are demonstrated in Figure 3. Only $15(17.4 \%)$ of the Arabic websites clarified the author of the presented content, and 11 (12.8\%) of the websites mentioned the data sources, such as journals, textbooks and guidelines.

More than half of the websites (64\%) presented the last date of update and $51(59 \%)$ of the websites disclosed "ownership" of the Web site With regard to the total number obtained in accordance with

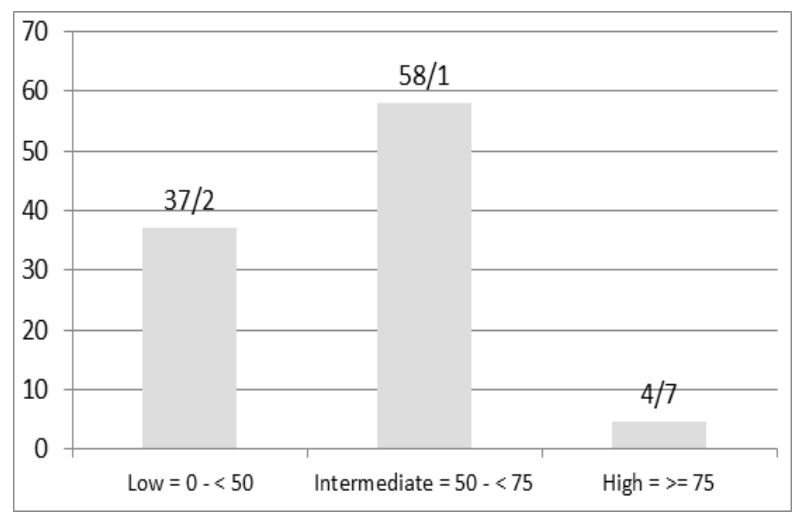

Figure 4. Shows Evaluation the Quality of the Medical Websites Using HON

JAMA benchmarks; four benchmarks were met only in 2 websites $(2.3 \%)$, three benchmarks met in 7 websites (8\%), two benchmarks met in 39 websites (45\%), one benchmark was met in 26 websites $(30 \%)$, and no benchmark was observed in 13 websites $(15 \%)$ (Supplementary Table 5). Therefore, 13 (15\%) of oral cancer related Arabic websites did not fulfill any criteria posed by the JAMA benchmarks and only $2(2 \%)$ of the sites fulfilled the four criteria (Figure 3).

\section{$H O N$}

The quality assessment of the 86 identified websites according to HON showed that only $4.7 \%$ of the sites achieved high score $(\geq 75)$ and $37.2 \%$ of the websites obtained low score $(<50)$, and $58 \%$ websites gained intermediate score $(\geq 50-<75)$ (Figure 4$)$.

Out of the 86 websites, $4(4.7 \%)$ achieved scores $>75 \%$ in $\mathrm{HON}$ assessment, of which three appeared in the top 10 based on the DISCERN assessment (Supplementary Table 7).

Table 2. Shows Evaluation the Quality of the Medical Websites Using DISCERN Assessment

\begin{tabular}{llcc}
\hline Domain & DESCREN Question & \multicolumn{2}{c}{ Score } \\
& & Mean (SD) & Median (IQR) \\
\hline Reliability & Q1 Explicit aim $(\mathrm{N}=85)$ & $2.68(1.39)$ & $2(1-4)$ \\
& Q2 Attainment of aims (N=81) & $2.05(0.92)$ & $2(1-3)$ \\
& Q3 Relevance $(\mathrm{N}=86)$ & $2.74(0.97)$ & $3(2-3)$ \\
& Q4 Explicit source $(\mathrm{N}=86)$ & $1.51(1.11)$ & $1(1-1)$ \\
& Q5 Explicit date $(\mathrm{N}=86)$ & $2.63(1.27)$ & $2(2-3)$ \\
& Q6 Balanced and Unbiased $(\mathrm{N}=86)$ & $2.8(1.03)$ & $2(1-3)$ \\
& Q7 Additional sources $(\mathrm{N}=85)$ & $1.96(0.96)$ & $3(2-4)$ \\
Qreatment Options & Q8 Areas of uncertainty $(\mathrm{N}=70)$ & $2.66(1.2)$ & $3(1-4)$ \\
& Q9 How treatment works $(\mathrm{N}=86)$ & $2.73(1.39)$ & $2.5(1-3)$ \\
& Q10 Benefits of treatment $(\mathrm{N}=86)$ & $1.99(1.22)$ & $1(1-3)$ \\
& Q11 Risk of treatment $(\mathrm{N}=86)$ & $2.14(1.1)$ & $2(1-3)$ \\
& Q12 Effects of no treatment $(\mathrm{N}=86)$ & $2.2(1.19)$ & $2(1-3)$ \\
& Q13 Effects on quality of life $(\mathrm{N}=86)$ & $2.39(1.29)$ & $2(1-3)$ \\
& Q14 All alternatives described $(\mathrm{N}=85)$ & $2.1(1.1)$ & $2(1-3)$ \\
& Q15 Shared decision (N=86) & $2.23(1.6)$ & $1(1-3)$ \\
\hline
\end{tabular}




\section{Discussion}

According to DISCERN, JAMA benchmarks, and HON, this study showed poor quality of information provided by oral cancer-related Arabic websites. This finding is in line with those reported by other studies about information provided on oral cancer at English, French, and Spanish websites (Saithna et al., 2008; López-Jornet and Camacho-Alonso, 2009; Irwin et al., 2011; Vivien et al., 2017). Patients are most likely to visit sites, which are listed in the first top 10 websites by search engines. Most of these search engines corporate with sponsors' links, that is, companies pay to be appeared at the top of the list for a particular search keyword, suggesting a bias towards information needed by patients from profit-making and therefore negative effect on the quality of provided data.

In this study, to overcome this limitation, most of profit and non-profit possible websites were included by browsing the first 100 websites and using 6 search engines. A non-profit organization is a group structured for a purpose other than making a profit, such as helping the community is concerned with money only as much as necessary to keep the organization operating. They can take the form of a corporation, partnership, an individual charitable contribution, or unincorporated association. they may include public hospitals and clinics, governmental agencies, political organizations, legal aid societies, volunteer service organizations, professional associations, and research institutes. A profit organization, as opposed to a non-profit one, aims at making money therefore, any Social forums contain any type of businesses whose primary goal is making money was classified as profit social websites and any Social forums including medical sites not contain trade advertisements or any business aim to making money was classified as nonprofit social websites (Frey, 2002).

Website failing to fulfill at least three of four criteria presented by JAMA benchmarks might be considered as doubtful (Silberg et al., 1997). According to the JAMA standards in this analysis, $90 \%$ of Arabic oral cancer sites were suspicious, which justifies the inability of $15 \%$ of Arab oral cancer sites in this study to ever meet any of the JAMA standards.

About $75 \%$ of the sites fulfilled two or three criteria of the JAMA benchmarks which more match the currency domain , and disclosure domain , contrary, the attribution domain achieved the lowest score in our assessment, following by the authorship domain as shown in JAMA benchmarks assessment (Figure 3), indicating that most of the Arabic websites did not mention the references and authorship, which were in line with results obtained by using DISCERN. However, the DISCERN assessment obtained a higher level score on the describing the treatment Q9 and the Explicit date Q5 (Table 2). Neglect to mention the references and authors at most of the Arabic oral cancer-related websites highly affected the quality of these websites. Therefore, it might then negatively influence the patients' ability to make proper and independent decisions.

In conclusion, findings of this study indicated that information on oral cancer is easily accessible at Arabic websites. However, a huge number of these websites were not adequately qualified for patient education. It is recommended to develop websites based system by which enable to the Arabic websites related to oral cancer know their shortfalls, therefore, improve their quality according to evaluation tools which will ensure finding reliable data from the websites.

\section{Acknowledgments}

We would like to express our special thanks to Dr Esam Halboub for cooperating with us in selecting and evaluating websites and conducting the statistical analysis.

\section{References}

Al-Ak'hali MS, Halboub ES, Mujam OH, et al (2017). Salivary parameters among Arabian snuff (Shammah) users. Saudi Med J, 38, 1201.

Al Huziah M, Al Kahtany M, Al Ammari R, et al (2009). Assessment of online health information for Arabic sites. MS Project Report. KSAU_HS.

Basha S, Mohamed RN, Al-Thomali Y, et al (2019). The prevalence of oral cancer in Saudi Arabia-A systematic review. Ann Med Health Sci Res, 9,553-7.

Boyer C, Baujard V, Geissbühler A (2011). Evolution of health web certification through the HONcode experience. Mie, 2011, 53-7.

Castleton K, Fong T, Wang-Gillam A, et al (2011). A survey of Internet utilization among patients with cancer. Supp Care Cancer, 19, 1183-90.

Charnock D, Shepperd S, Needham G, et al (1999). DISCERN: an instrument for judging the quality of written consumer health information on treatment choices. $J$ Epidemiol Community Health, 53, 105-11.

Ferlay J, Soerjomataram I, Dikshit R, et al (2015). Cancer incidence and mortality worldwide: sources, methods and major patterns in GLOBOCAN 2012. Int $J$ Cancer, 136, $359-86$.

Frey JC (2002). Guidebook for directors of nonprofit corporations. 2002. American Bar Association.

Irwin JY, Thyvalikakath T, Spallek H, et al (2011). English and Spanish oral cancer information on the internet: a pilot surface quality and content evaluation of oral cancer web sites. J Public Health Dent, 71, 106-16.

Jamal A, Khan SA, AlHumud A, et al (2015). Association of online health information-seeking behavior and self-care activities among type 2 diabetic patients in Saudi Arabia. $J$ Med Int Res, 17, e196.

Lebbos G, Zreik K, El-Sayed M (2014). Performances of the most popular search engines in Arabic language. Int $J$ Comput Theory Eng, 6, 4.

López-Jornet P, Camacho-Alonso F (2009). The quality of internet sites providing information relating to oral cancer. Oral Oncol, 45, 95-8.

Organization WH, Organization WH (2009). Regional office for the Eastern Mediterranean. E-Health in the Eastern Mediterranean. URL: http://208.48, 48.

Portnoy DB, Scott-Sheldon LA, Johnson BT, et al (2008). Computer-delivered interventions for health promotion and behavioral risk reduction: a meta-analysis of 75 randomized controlled trials, 1988-2007. Prev Med, 47, 3-16.

Saithna A, Ajayi O, Davis E (2008). The quality of Internet sites providing information relating to hip resurfacing. Surgeon, 6, 85-7.

Schwarzer R, Fuchs R (1995). Changing risk behaviors and

Asian Pacific Journal of Cancer Prevention, Vol 21 
adopting health behaviors: The role of self-efficacy beliefs. Self-efficacy in changing societies, 259, pp 288.

Silberg WM, Lundberg GD, Musacchio RA (1997). Assessing, controlling, and assuring the quality of medical information on the Internet: Caveant lector et viewor-Let the reader and viewer beware. JAMA, 277, 1244-5.

Strecher VJ (1999). Computer-tailored smoking cessation materials: a review and discussion. Patient Educ Couns, 36, 107-7.

Vivien A, Kowalski V, Chatellier A, et al (2017). Information quality in general public French-speaking websites dedicated to oral cancer detection. J Oral Maxillofac Surg, 118, 20-8.

Warnakulasuriya S (2009). Global epidemiology of oral and oropharyngeal cancer. Oral Oncol, 45, 309-16.

Warnakulasuriya S (2010). Living with oral cancer: epidemiology with particular reference to prevalence and life-style changes that influence survival. Oral Oncol, 46, 407-10.

\section{๑.⿴囗十}

This work is licensed under a Creative Commons AttributionNon Commercial 4.0 International License. 